\title{
Adjacent SNPs in the transcriptional regulatory region of the $F A D S 2$ gene associated with fatty acid and growth traits in chickens
}

\author{
S.K. Zhu ${ }^{1 *}$, Y.D. Tian ${ }^{1,2 *}$, S. Zhang ${ }^{1}$, Q.X. Chen ${ }^{1,2}$, Q.Y.Wang ${ }^{3}$, \\ R.L. Han ${ }^{1,2}$ and X.T. Kang ${ }^{1,2}$ \\ ${ }^{1}$ College of Animal Science and Veterinary Medicine, \\ Henan Agricultural University, Zhengzhou, China \\ ${ }^{2}$ Henan Innovative Engineering Research Center of Poultry Germplasm Resource, \\ Zhengzhou, China \\ ${ }^{3}$ Xuchang University, Xuchang, China \\ *These authors contributed equally to this study. \\ Corresponding author: X.T. Kang \\ E-mail: xtkang2001@263.net
}

Genet. Mol. Res. 13 (2): 3329-3336 (2014)

Received January 28, 2013

Accepted June 20, 2013

Published April 29, 2014

DOI http://dx.doi.org/10.4238/2014.April.29.11

\begin{abstract}
Delta-6 fatty acid desaturases are rate-limiting desaturases involved in metabolic processes of fatty acids, and they are encoded by the FADS 2 gene. In the current study, an $\mathrm{F}_{2}$ resource population of Gushi chickens crossed with Anak broilers was used to investigate the genetic effects of the chicken FADS2. Two adjacent single nucleotide polymorphisms (SNPs) (g.4290C $>$ G and g.4291C $>$ A) were identified in the transcriptional regulatory region of the FADS2 gene by means of polymerase chain reaction-restriction fragment length polymorphism (PCR-RFLP) and created restriction site-PCR-RFLP. Associations between the two SNPs with chicken fatty acid contents and growth traits were determined using linkage disequilibrium, haplotype construction, and association analysis. The two SNPs and their haplotype combinations were significantly associated with linoleic
\end{abstract}


acid (C18:2), $\alpha$-linolenic acid (C18:3), arachidonic acid (C20:4), body weight (BW)2, BW4, BW6, shank girth (SG)4, and breast bone length $4(\mathrm{P}<0.05)$. These results suggested that the SNPs of the FADS2 gene affected the content of essential fatty acid in muscle, and played a role in the early-stage growth rate of chickens.

Key words: Chicken; FADS2; Single nucleotide polymorphisms; Haplotype; Fatty acid; Growth traits

\section{INTRODUCTION}

Delta-6 desaturase catalyzes linoleic acid (LA, C18:2) and $\alpha$-linolenic acid (ALA, $\mathrm{C} 18: 3)$ to $\gamma$-linolenic acid (GLA, C18:3) and stearidonic acid (C18:4), which involves the insertion of a double bond between carbon 6 and 7 by dehydrogenation. It is generally accepted that the delta-6 desaturation step is rate-limiting (Gill and Valivety, 1997; Kohamaet al., 1998; Innis, 2003; Vance and Vance, 2008). Subsequently, GLA (C18:3) and stearidonic acid (C18:4) could be further biosynthesized into the long-chain polyunsaturated fatty acids (LCPUFAs) arachidonic acid (C20:4), eicosapentaenoic acid (EPA, C20:5), and docosahexaenoic acid (DHA, C22:6), as a result of further desaturase dehydrogenations (delta-5 desaturase) and elongase steps (Gill and Valivety, 1997; Nakamura and Nara, 2004; Baylin et al., 2007). These LC-PUFAs are important components for the maintenance of normal cell function and for increasing the body's resistance of the neonates (Farrellet al., 1988). Delta-6 desaturase is encoded by the delta-6 fatty acid desaturases (FADS) gene, FADS2. Studies have found that single nucleotide polymorphisms (SNPs) located on the FADS gene cluster contributed to the variability of several PUFAs, such as LA, ALA, and eicosadienoic acids, especially arachidonic acid in serum phospholipids and erythrocyte cell membranes (Malerba et al., 2008). Nwankwo et al. (2003) demonstrated that a nucleotide insertion in the transcriptional regulatory region of the human FADS2 gene resulted in delta-6 desaturase deficiency and decreased FADS2 transcription. Further evidence showed that a FADS2 promoter polymorphism increased promoter activity and facilitated binding of the transcription factor ELK1 (Lattka et al., 2010). Furthermore, an SNP in the FADS1/FADS2 gene appeared to cause a change in plasma lipid profiles in two genetically similar Asian ethnic groups with distinctive lifestyle differences (Nakayamaet al., 2010). Research related to the FADS2 gene in poultry is more limited, however. Khang et al. (2006) investigated genetic variation in the FADS1 and FADS2 genes among different local Vietnamese chicken breeds, and found no polymorphisms in FADS2. This study also identified five SNPs and an association was found between these polymorphisms and the egg yolk fatty acid composition in the FADS2 gene of Japanese quail. This study therefore suggested FADS2 as a functional candidate gene for traits related to n- 6 and n-3PUFA concentration in eggs, as demonstrated in experimental quail selection lines (Khang et al., 2007). However, it is currently not known whether FADS2 plays a role in chicken growth and development. The FADS2 gene contains 12 exons and 11 introns, and was clustered with family member FADS1 on chromosome 5 in chicken, while in humans it clusters with FADS1 and FADS3 on chromosome 11. The first exon and intron are the longest (Cho et al., 1999; Khang et al., 2007; Kang et al., 2010). In the present study, we identified genetic variations in chicken FADS2 through DNA pool sequencing, polymerase chain reaction-restriction fragment length polymorphism 
(PCR-RFLP) and created restriction site (CRS)-PCR-RFLP methods, and analyzed relationships between genetic variations and some growth traits in chickens. The objectives of this study were to identify genetic polymorphisms in the FADS2 gene and to elucidate their associations with chicken performance traits.

\section{MATERIAL AND METHODS}

\section{Resource populations}

In this study, we used an $\mathrm{F}_{2}$ resource family, whose details have been described in a previous study (Han et al., 2011). Seventy $\mathrm{F}_{1}$ animals ( 7 males, 63 females) were produced from Gushi chickens ( 24 hens and 2 roosters) and Anak broilers ( 12 hens and 4 roosters). Four Anak male chickens were mated with 24 Gushi females and 2 Gushi males were mated to 12 Anak females. A total of 860 offspring were produced from the $F_{1}$ population, which formed the $F_{2}$ Gushi-Anak resource population. All chickens were reared under the same environmental conditions with free access to feed and water.

\section{Measurement of phenotypes}

Growth traits of chickens were analyzed in this study, along with body weight (BW) and size. The $860 \mathrm{~F}_{2}$ chickens were weighed individually at $0,2,4,6,8,10$, and 12 weeks respectively, while body size was measured at $0,4,8$, and 12 weeks, including shank length (SL), shank girth (SG), chest depth (CD), chest breadth (CB), breast bone length (BBL), pectoral angle (PA), pelvis breadth (PB), and body slanting length (BSL). All chickens were slaughtered after 12 weeks. Additionally, the fatty acid constituents of muscle were separated and identified by gas chromatography-mass spectrometry (GC-MS), of which myristic acid (C14:0), palmitic acid (C16:0), palmitoleic acid (C16:1), stearic acid (C18:0), oleic acid (C18:1), LA (C18:2), ALA (C18:3), eicosadienoic acid (C20:2), arachidonic acid (C20:4), EPA (C20:5), and DHA (C22:6) were detected.

\section{DNA samples, PCR conditions, and genotyping}

Genomic DNA samples were extracted from plasma of $\mathrm{F}_{2}$ resource populations by the phenol-chloroform method. More than 150 DNA samples from $\mathrm{F}_{2}$ individuals were randomly selected to construct the DNA pool, which was sent to Taihegene Biotechnology Co., Ltd (Beijing, China) for SNP screening of the FADS2 gene.

Two pairs of primers were designed based on the sequencing results and the chicken FADS2 sequence (GenBank: NW_001471700). Primer P1 (F: 5'-TGAACTGAAGCGTTTGATGG-3'， R: 5'-TGGCTTTCTTGGCAATAGG-3') was designed to amplify the 283-bp fragment containing g.4290C $>\mathrm{G}$ in the promoter region of the FADS2 gene. Meanwhile, P2 (F: 5'-ATGGTCAGCCATAGGTCTCCTTT-3', R: 5'-GGCTTCACTCACTGATGTTACTA-3') was designed for the 167-bp fragment covering the g.4291C $>$ A, which was amplified by created restriction site (CRS)-PCR-RFLP. All primers were provided by Sangon Biotech (Shanghai) Co Ltd (Shanghai, China).

The PCR amplification system was performed in a total volume of $25 \mu \mathrm{L}$, containing 
50 ng DNA, $1 \mu \mathrm{M}$ each primer, and $12 \mu \mathrm{L}$ Taq MasterMix (Cwbiotech, Beijing). The PCR was run according to the following program: $5 \mathrm{~min}$ at $95^{\circ} \mathrm{C}$ (initial denaturation), 30 cycles of $94^{\circ} \mathrm{C}$ for $30 \mathrm{~s}, 56.5^{\circ} \mathrm{C}$ (primer $\mathrm{P} 1$ ) or $60^{\circ} \mathrm{C}$ (primer P2) for $30 \mathrm{~s}$, and $72^{\circ} \mathrm{C}$ for $30 \mathrm{~s}$, and a final extension at $72^{\circ} \mathrm{C}$ for $10 \mathrm{~min}$.

The PCR products were digested by restriction enzyme ( $R s a$ I for g.4290C $>\mathrm{G}$ and SpeI for g. $4291 \mathrm{C}>\mathrm{A}$ ) overnight at $37^{\circ} \mathrm{C}$. Finally, the digestion products were separated by electrophoresis on $1.5 \%($ g. $4290 \mathrm{C}>\mathrm{G})$ and $3 \%(\mathrm{~g} .4291 \mathrm{C}>\mathrm{A})$ agarose gels with ethidium bromide staining. Samples of each genotype were selected and sequenced by Sangon Biotech (Shanghai) Co Ltd. (Shanghai, China).

\section{Linkage disequilibrium and haplotype}

Linkage disequilibrium (LD) between two SNPs was estimated as pairwise $\mathrm{r}^{2}$ and D' values using the SHEsis program (http://analysis.bio-x.cn/myAnalysis.php). Individual haplotypes were inferred by the use of the two SNPs and the PHASE software package version 2.1 (Shi and He, 2005). Haplotypes with frequencies greater than $1 \%$ were retained for further consideration.

\section{Statistical analysis}

Phenotypic correlations between genotypes and the selected traits of $\mathrm{F}_{2}$ chickens were calculated using the following linear mixed model in SPSS 17.0:

$$
\mathrm{Y}_{\mathrm{ijklm}}=\mu+\mathrm{G}_{\mathrm{i}}+\mathrm{S}_{\mathrm{j}}+\mathrm{H}_{\mathrm{k}}+\mathrm{f}_{1}+\mathrm{e}_{\mathrm{ijklm}}
$$

where $Y_{i j k l m}$ is the dependent variable (analyzed traits), $\mu$ is the overall mean, $G_{i}$ is the fixed effect of genotype or haplotype, $S_{j}$ is the fixed effect of sex, $H_{k}$ is the fixed effect of hatch, $f_{l}$ is the random effect of family, and $e_{i j k l m}$ is the random error.

The effect of genotypes or haplotypes of the polymorphisms of the FADS2 gene on target traits were investigated by least-squares analysis. In cases where the effect of genotypes or haplotypes was significant $(\mathrm{P}<0.05)$, the Bonferroni test was used for multiple comparisons of the genotypes, and the additive and dominance effects were estimated using linear regression in SPSS 17.0. The additive effect was estimated as 1, 0, and -1, representing the $\mathrm{CC}(\mathrm{AA}), \mathrm{GC}(\mathrm{AC})$, and $\mathrm{GG}(\mathrm{CC})$ genotypes, respectively, whereas the dominance effect was estimated as $1,-1$, and 1 , representing the $\mathrm{CC}(\mathrm{AA}), \mathrm{GC}(\mathrm{AT})$, and $\mathrm{GG}(\mathrm{CC})$ genotypes, respectively.

\section{RESULTS}

\section{SNP identification and genotyping}

DNA sequencing identified two adjacent novel SNPs, g.4290C $>$ G and g.4291C $>$ A, in the transcriptional regulatory region of the FADS2 gene. For both SNPs, three genotypes were detected through the (CRS)-PCR-RFLP method, which were confirmed by sequencing the homozygote PCR products. For the g. $4290 \mathrm{C}>\mathrm{G}$ site, the frequencies of alleles $\mathrm{C}$ and $\mathrm{G}$ 
were 0.632 and 0.368 , respectively. The genotype frequencies of CC, GC, and GG were 0.377 , 0.512 , and 0.112 , respectively. For g.4291C $>\mathrm{A}$, the allele frequencies of $\mathrm{C}$ and $\mathrm{A}$ were 0.546 and 0.454 , respectively, and the genotype frequencies of $\mathrm{CC}, \mathrm{AC}$, and $\mathrm{AA}$ were $0.230,0.632$, and 0.138 , respectively.

\section{Linkage disequilibrium and haplotype construction}

The two SNPs were in strong LD, which was estimated as $\mathrm{D}^{\prime}=0.975, \mathrm{r}^{2}=0.459$. Through estimating SNP haplotype frequencies by using PHASE 2.0, three haplotypes were observed with frequencies $>0.01$ among individuals of the $\mathrm{F}_{2}$ resource population, including H1 (C-A), H2 (C-C), and H4 (G-C), with frequencies of $0.450,0.183$, and 0.363 , respectively; H3 (G-A) was deleted from further analysis due to its low frequency (0.004).

\section{Associations of SNPs and chicken performance traits}

The g.4290C $>$ G polymorphism showed significant associations with BW2, BW4, BW6, SL4, SG4, BBL4, ALA (C18:3), and arachidonic acid (C20:4) $(\mathrm{P}<0.05)$. Furthermore, chickens with the GC genotype had higher SL4 values. For the other traits, the GG genotype showed significantly higher values than did the GC and $\mathrm{CC}$ genotypes. In tests for additive and dominant effects, additive effects were significant $(\mathrm{P}<0.05)$ for BBL4 and ALA $(\mathrm{C} 18: 3)$, and the dominance effect was significant $(\mathrm{P}<0.05)$ for arachidonic acid $(\mathrm{C} 20: 4)$.

Significant associations for the g.4291C $>$ A polymorphism were found with birth body weight (BBW), BW2, BW4, BW6, SG4, BBL4, LA (C18:2), and arachidonic acid (C20:4) (P < $0.05)$. The mean values of these traits were significantly higher in genotype $C C$ than in genotypes CA and AA. For BW4, BW6, BBL4, and LA (C18:2), the additive effects were significant $(\mathrm{P}<$ $0.05)$, and the dominance effect was significant $(\mathrm{P}<0.05)$ for arachidonic acid $(\mathrm{C} 20: 4)$ (Table 1).

\begin{tabular}{|c|c|c|c|c|c|c|c|c|c|}
\hline \multirow[t]{2}{*}{ SNPs } & \multirow[t]{3}{*}{ Traits } & \multicolumn{3}{|c|}{ SNP genotype } & \multirow[t]{3}{*}{$\mathrm{P}$} & \multirow{3}{*}{$\frac{\text { Additive effect }}{\mathrm{B} \pm \mathrm{SE}}$} & \multirow[t]{3}{*}{$P$} & Dominance effect & \multirow[t]{3}{*}{$\mathrm{P}$} \\
\hline & & \multicolumn{3}{|c|}{ Means \pm SE } & & & & $\mathrm{B} \pm \mathrm{SE}$ & \\
\hline \multirow[t]{9}{*}{$\underline{\text { g. } 4290 \mathrm{C}>\mathrm{G}}$} & & $\mathrm{CC}$ & $\mathrm{GC}$ & GG & & & & & \\
\hline & BW2 (g) & $121.352 \pm 2.499^{\mathrm{a}}$ & $122.622 \pm 2.423$ & $127.378 \pm 3.022^{\mathrm{b}}$ & 0.037 & $-0.048 \pm 1.028$ & 0.963 & $0.547 \pm 0.669$ & 0.414 \\
\hline & BW4 (g) & $315.536 \pm 7.612^{\mathrm{a}}$ & $322.827 \pm 7.466$ & $334.295 \pm 8.683^{\mathrm{b}}$ & 0.003 & $-2.491 \pm 2.488$ & 0.317 & $0.075 \pm 1.612$ & 0.963 \\
\hline & BW6 (g) & $554.757 \pm 14.182^{\mathrm{a}}$ & $560.482 \pm 13.912$ & $582.516 \pm 16.254^{b}$ & 0.037 & $-3.394 \pm 4.706$ & 0.471 & $2.801 \pm 3.056$ & 0.360 \\
\hline & SL4 (cm) & $5.472 \pm 0.071$ & $5.634 \pm 0.067^{\mathrm{b}}$ & $5.322 \pm 0.098^{\mathrm{a}}$ & 0.041 & $0.072 \pm 0.040$ & 0.071 & $-0.034 \pm 0.026$ & 0.193 \\
\hline & $\mathrm{SG} 4(\mathrm{~cm})$ & $2.683 \pm 0.030^{\mathrm{a}}$ & $2.700 \pm 0.029$ & $2.748 \pm 0.035^{b}$ & 0.038 & $0.010 \pm 0.011$ & 0.385 & $0.007 \pm 0.007$ & 0.360 \\
\hline & BBL4 $(\mathrm{cm})$ & $6.147 \pm 0.064^{\mathrm{a}}$ & $6.234 \pm 0.062$ & $6.389 \pm 0.080^{\mathrm{b}}$ & 0.001 & $-0.057 \pm 0.027$ & 0.040 & $-0.008 \pm 0.018$ & 0.669 \\
\hline & $\mathrm{C} 18-3$ & $0.178 \pm 0.019^{\mathrm{a}}$ & $0.193 \pm 0.015$ & $0.294 \pm 0.033^{b}$ & 0.008 & $-0.044 \pm 0.017$ & 0.012 & $0.006 \pm 0.011$ & 0.580 \\
\hline & $\mathrm{C} 20-4$ & $2.248 \pm 0.108$ & $2.033 \pm 0.086^{\mathrm{a}}$ & $2.897 \pm 0.191^{\mathrm{b}}$ & 0.000 & $-0.159 \pm 0.103$ & 0.126 & $0.183 \pm 0.065$ & 0.005 \\
\hline \multirow[t]{9}{*}{ g. $4291 \mathrm{C}>\mathrm{A}$} & & AA & $\mathrm{AC}$ & $\mathrm{CC}$ & & & & & \\
\hline & BBW (g) & $30.068 \pm 0.524^{a}$ & $30.576 \pm 0.462$ & $31.061 \pm 0.496^{b}$ & 0.017 & $-0.189 \pm 0171$ & 0.269 & $-0.173 \pm 0.108$ & $\overline{0.112}$ \\
\hline & BW2 (g) & $118.742 \pm 2.894^{\mathrm{a}}$ & $121.924 \pm 2.411^{\mathrm{a}}$ & $126.421 \pm 2.670^{\mathrm{b}}$ & 0.002 & $-1.880 \pm 1.109$ & 0.090 & $0.124 \pm 0.702$ & 0.860 \\
\hline & BW4 (g) & $311.473 \pm 7.920^{\mathrm{a}}$ & $320.569 \pm 6.896$ & $328.985 \pm 7.437^{b}$ & 0.006 & $-6.105 \pm 2.649$ & 0.021 & $0.796 \pm 1.684$ & 0.637 \\
\hline & BW6 (g) & $543.404 \pm 15.274^{a}$ & $560.466 \pm 13.235$ & $570.675 \pm 14.277^{b}$ & 0.037 & $-10.351 \pm 5.072$ & 0.042 & $1.785 \pm 3.213$ & 0.579 \\
\hline & $\mathrm{SG} 4(\mathrm{~cm})$ & $2.658 \pm 0.032^{\mathrm{a}}$ & $2.696 \pm 0.027$ & $2.726 \pm 0.030^{\mathrm{b}}$ & 0.022 & $-0.016 \pm 0.012$ & 0.180 & $0.002 \pm 0.008$ & 0.823 \\
\hline & BBL4 $(\mathrm{cm})$ & $6.134 \pm 0.071^{\mathrm{a}}$ & $6.203 \pm 0.055$ & $6.312 \pm 0.064^{b}$ & 0.009 & $-0.066 \pm 0.029$ & 0.026 & $0.011 \pm 0.019$ & 0.555 \\
\hline & $\mathrm{C} 18-2$ & $2.539 \pm 0.193^{\mathrm{a}}$ & $2.569 \pm 0.091$ & $3.032 \pm 0.156^{\mathrm{b}}$ & 0.030 & $-0.285 \pm 0.123$ & 0.021 & $0.134 \pm 0.078$ & 0.085 \\
\hline & $\mathrm{C} 20-4$ & $2.350 \pm 0.170$ & $2.053 \pm 0.080^{\mathrm{a}}$ & $2.541 \pm 0.137^{b}$ & 0.006 & $-0.158 \pm 0.110$ & 0.151 & $0.200 \pm 0.068$ & 0.004 \\
\hline
\end{tabular}

${ }_{a, b}$ Means within a row with no common superscript differ significantly $(\mathrm{P}<0.05)$. 


\section{Associations of the haplotypes with chicken growth and performance traits}

Six haplotype combinations were found in the $\mathrm{F}_{2}$ resource population. Significant associations were found between the haplotype combinations and LA (C18:2), ALA (C18:3), arachidonic acid (C20:4), BW2, BW4, BW6, SG4, and BBL4 $(\mathrm{P}<0.05)$. For most of these, the diplotype of H4H4 (G-C/G-C) showed significantly higher levels than those with other diplotypes (Table 2).

\begin{tabular}{|c|c|c|c|c|c|c|c|}
\hline \multirow[t]{3}{*}{ Traits } & \multicolumn{6}{|c|}{ Haplotype combinations } & \multirow[t]{3}{*}{$\mathrm{P}$} \\
\hline & \multicolumn{6}{|c|}{ Means $\pm \mathrm{SE}$} & \\
\hline & H1H1 & $\mathrm{H} 1 \mathrm{H} 2$ & $\mathrm{H} 1 \mathrm{H} 4$ & $\mathrm{H} 2 \mathrm{H} 2$ & $\mathrm{H} 2 \mathrm{H} 4$ & $\mathrm{H} 4 \mathrm{H} 4$ & \\
\hline BW2(g) & $118.885 \pm 2.912^{\mathrm{a}}$ & $122.748 \pm 2.665$ & $121.626 \pm 2.476$ & $122.851 \pm 5.308$ & $127.201 \pm 3.100^{\mathrm{b}}$ & $127.380 \pm 3.039^{b}$ & 0.011 \\
\hline BW4(g) & $311.253 \pm 8.294^{\mathrm{a}}$ & $320.524 \pm 7.827$ & $320.971 \pm 7.419$ & $315.424 \pm 12.923$ & $332.378 \pm 8.681^{\mathrm{b}}$ & $333.842 \pm 8.601^{\mathrm{b}}$ & 0.008 \\
\hline BW6(g) & $542.898 \pm 16.218^{\mathrm{a}}$ & $562.878 \pm 15.136$ & $558.612 \pm 14.457$ & $555.993 \pm 25.221$ & $572.883 \pm 16.906$ & $587.333 \pm 16.642^{\mathrm{b}}$ & 0.019 \\
\hline $\mathrm{SG} 4(\mathrm{~cm})$ & $2.659 \pm 0.033$ & $2.707 \pm 0.031$ & $2.693 \pm 0.029$ & $2.680 \pm 0.055$ & $2.733 \pm 0.035$ & $2.746 \pm 0.035$ & 0.045 \\
\hline $\mathrm{BBL} 4(\mathrm{~cm})$ & $6.133 \pm 0.076^{\mathrm{a}}$ & $6.178 \pm 0.069$ & $6.216 \pm 0.063$ & $6.157 \pm 0.133$ & $6.332 \pm 0.081$ & $6.362 \pm 0.080^{\mathrm{b}}$ & 0.014 \\
\hline $\mathrm{C} 18-2$ & $2.539 \pm 0.192^{\mathrm{a}}$ & $2.453 \pm 0.170^{\mathrm{a}}$ & $2.624 \pm 0.107^{\mathrm{a}}$ & $4.221 \pm 0.486^{\mathrm{b}}$ & $2.972 \pm 0.243$ & $2.794 \pm 0.217$ & 0.015 \\
\hline C18-3 & $0.225 \pm 0.029$ & $0.146 \pm 0.026^{\mathrm{a}}$ & $0.198 \pm 0.016$ & $0.140 \pm 0.073$ & $0.174 \pm 0.037$ & $0.287 \pm 0.033^{\mathrm{b}}$ & 0.021 \\
\hline C20-4 & $2.350 \pm 0.169$ & $2.177 \pm 0.150$ & $2.008 \pm 0.094^{\mathrm{a}}$ & $2.024 \pm 0.428$ & $2.225 \pm 0.214$ & $2.870 \pm 0.191^{\mathrm{b}}$ & 0.005 \\
\hline
\end{tabular}

a,b Means within a row with no common superscript differ significantly $(\mathrm{P}<0.05)$.

\section{DISCUSSION}

This study was the first to evaluate associations between genetic variants in the transcriptional regulatory region of the FADS2 gene with fatty acids in a poultry resource family. In this study, two adjacent novel SNPs were found in the transcriptional regulatory region of the FADS2 gene. We evaluated whether SNPs in the FADS2 gene were associated with chicken performance traits. Furthermore, owing to the two SNPs being in close LD, we constructed the haplotypes and then analyzed whether the haplotype combinations showed similar results. The results revealed that the two SNPs and their haplotype combinations showed significant associations with some performance traits, such as LA (C18:2), ALA (C18:3), arachidonic acid (C20:4), BW2, BW4, BW6, SG4, and BBL4.

Until now, the majority of the research concerning FADS2 has focused on humans, which demonstrated that the FADS1/FADS2 loci were associated with plasma triglyceride, HDL-C, and LDL-C concentrations in populations with European ancestry (Kathiresan et al., 2008; Aulchenko et al., 2009). Polymorphisms of FADS1/FADS2 showed strongest associations with fatty acid levels such as LA (C18:2), g-linolenic acid (C18:3), eicosadienoic acid (C20:2), arachidonic acid (C20:4), ALA (C18:3), dihomo-g-linolenic acid (C20:3), adrenic acid (C22:4), eicosapentaenoic acid (C20:5), docosapentaenoic acid (C22:5), among others in human serum phospholipids (Schaeffer et al., 2006). Similar results were demonstrated in the present study on poultry. The two adjacent novel SNPs of the FADS2 gene, as well as their corresponding haplotypes, were most highly associated with LA (C18:2), ALA (C18:3), and arachidonic acid (C20:4), which are essential fatty acids of the chicken. LA (C18:2) and ALA (C18:3) cannot be biosynthesized de novo, and are instead derived from the diet, and subsequently cannot be bioconverted to longer and more unsaturated LC-PUFAs. Further- 
more, arachidonic acid (C20:4) was found to be the major metabolite of dietary LA (C18:2) (Poureslami et al., 2010). Merino et al. (2011) reported that genetic variation in the FADS gene cluster could alter desaturase activity in human subjects of Caucasian and Asian descent. In the present study, the data was insufficient to determine whether the two adjacent mutations could result in enzyme activity; therefore, the mechanism that caused the observed changes in fatty acid content require further validation.

In poultry, five SNPs were identified in Japanese quail and an association was found between these polymorphisms and the egg yolk fatty acid composition (Khang et al., 2007). However, reports investigating the associations between growth traits and SNPs of the FADS2 gene in poultry are scarce. The current study provides strong evidence that the adjacent SNPs and their haplotype combinations are associated with BW2, BW4, BW6, SG4, and BBL4. Therefore, the SNPs were associated with the early-stage growth rate of chickens, especially at 4 weeks. In the later growth stage, this advantage became negligible $(\mathrm{P}>0.05)$.

In chicken breeding practices, some chickens are selected on the basis of precocity and their ability to reach their market live weight at an early age. The combination of age, low kinetic activity, and high feed intake results in a higher fat accumulation in muscles (Dal et al., 2012). However, in our study, the fast growth rate did not cause significant accumulation of fat in muscles (data not shown). This trait combination would be beneficial for the selection and breeding of high quality broiler chicken species. We speculated that this mutation caused the appearance of the aforementioned fatty acids and its accumulation in the muscle, which would affect the early-stage growth rate of chickens; however, the precise mechanism requires further investigation.

In conclusion, two adjacent SNPs were found in the transcriptional regulatory region of the FADS2 gene. Both SNPs showed significant associations with body weight and fatty acid traits. Therefore, these results support the hypothesis that the FADS2 gene plays an important role in the early-stage growth rate of chickens. The limitations of the present study should be considered when interpreting these results and the specific desaturase activity should be measured in future related studies.

\section{ACKNOWLEDGMENTS}

Research supported by the National Natural Science Foundation of China (\#31072023), the Foundation for Development Program of Innovation Team from the Ministry of Education of China (\#IRT1236), and the Earmarked Fund for Modern Agro-industry Technology Research System (\#CARS-41-K04).

\section{REFERENCES}

Aulchenko YS, Ripatti S, Lindqvist I, Boomsma D, et al. (2009). Loci influencing lipid levels and coronary heart disease risk in 16 European population cohorts. Nat. Genet. 41: 47-55.

Baylin A, Ruiz-Narvaez E, Kraft P and Campos H (2007). alpha-Linolenic acid, Delta6-desaturase gene polymorphism, and the risk of nonfatal myocardial infarction. Am. J. Clin. Nutr. 85: 554-560.

Cho HP, Nakamura MT and Clarke SD (1999). Cloning, expression, and nutritional regulation of the mammalian Delta-6 desaturase. J. Biol. Chem. 274: 471-477.

Dal BA, Mugnai C, Ruggeri S, Mattioli S, et al. (2012). Fatty acid composition of meat and estimated indices of lipid metabolism in different poultry genotypes reared under organic system. Poult. Sci. 91: 2039-2045.

Farrell PM, Gutcher GR, Palta M and DeMets D (1988). Essential fatty acid deficiency in premature infants. Am. J. Clin. 
Nutr. 48: 220-229.

Gill I and Valivety R (1997). Polyunsaturated fatty acids, Part 1: Occurrence, biological activities and applications. Trends Biotechnol. 15: 401-409.

Han RL, Li ZJ, Li MJ, Li JQ, et al. (2011). Novel 9-bp indel in visfatin gene and its associations with chicken growth. $B r$. Poult. Sci. 52: 52-57.

Innis SM (2003). Perinatal biochemistry and physiology of long-chain polyunsaturated fatty acids. J. Pediatr. 143: S1-S8.

Kang XT, Bai YC, Sun GR, Huang YQ, et al. (2010). Molecular cloning, characterization, and expression analysis of chicken Delta-6 desaturase. Asian Austral J. Anim. 23: 116-121.

Kathiresan S, Melander O, Guiducci C, Surti A, et al. (2008). Six new loci associated with blood low-density lipoprotein cholesterol, high-density lipoprotein cholesterol or triglycerides in humans. Nat. Genet. 40: 189-197.

Khang NT, Jennen DG, Mennicken L, Tesfaye D, et al (2006). Genetic Variety of the Different Vietnamese Local Chicken Breeds and Effect on the Fads1 and Fads2 Genes. Tropical and Subtropical Agricultural and Natural Resource Management, Bonn.

Khang NT, Jennen DG, Tholen E, Tesfaye D, et al. (2007). Association of the FADS2 gene with omega-6 and omega-3 PUFA concentration in the egg yolk of Japanese quail. Anim. Biotechnol. 18: 189-201.

Kohama T, Olivera A, Edsall L, Nagiec MM, et al. (1998). Molecular cloning and functional characterization of murine sphingosine kinase. J. Biol. Chem. 273: 23722-23728.

Lattka E, Eggers S, Moeller G, Heim K, et al. (2010). A common FADS2 promoter polymorphism increases promoter activity and facilitates binding of transcription factor ELK1. J. Lipid. Res. 51: 182-191.

Malerba G, Schaeffer L, Xumerle L, Klopp N, et al. (2008). SNPs of the FADS gene cluster are associated with polyunsaturated fatty acids in a cohort of patients with cardiovascular disease. Lipids 43: 289-299.

Merino DM, Johnston H, Clarke S, Roke K, et al. (2011). Polymorphisms in FADS1 and FADS2 alter desaturase activity in young Caucasian and Asian adults. Mol. Genet. Metab. 103: 171-178.

Nakamura MT and Nara TY (2004). Structure, function, and dietary regulation of delta6, delta5, and delta9 desaturases. Annu. Rev. Nutr. 24: 345-376.

Nakayama K, Bayasgalan T, Tazoe F, Yanagisawa Y, et al. (2010). A single nucleotide polymorphism in the FADS1/ FADS2 gene is associated with plasma lipid profiles in two genetically similar Asian ethnic groups with distinctive differences in lifestyle. Hum. Genet. 127: 685-690.

Nwankwo JO, Spector AA and Domann FE (2003). A nucleotide insertion in the transcriptional regulatory region of FADS2 gives rise to human fatty acid delta-6-desaturase deficiency. J. Lipid. Res. 44: 2311-2319.

Poureslami R, Raes K, Turchini GM, Huyghebaert G, et al. (2010). Effect of diet, sex and age on fatty acid metabolism in broiler chickens: n-3 and n-6 PUFA. Br. J. Nutr. 104: 189-197.

Schaeffer L, Gohlke H, Muller M, Heid IM, et al. (2006). Common genetic variants of the FADS1 FADS2 gene cluster and their reconstructed haplotypes are associated with the fatty acid composition in phospholipids. Hum. Mol. Genet. 15: $1745-1756$

Shi YY and He L (2005). SHEsis, a powerful software platform for analyses of linkage disequilibrium, haplotype construction, and genetic association at polymorphism loci. Cell Res. 15: 97-98.

Vance JE and Vance DE (2008). Biochemistry of Lipids, Lipoproteins and Membranes. 5th edn. Elsevier Science Ltd., Canada. 